PALEO

Revue d'archéologie préhistorique

25 | 2014

Varia

\title{
Actualisation de l'inventaire des pointes de type Isturitz de la région cantabrique
}

An updated inventory of the Isturitz type bone points of the cantabrian region

Joseba Rios-Garaizar et Diego Garate

\section{OpenEdition}

1 Journals

Édition électronique

URL : http://journals.openedition.org/paleo/2811

DOI : $10.4000 /$ paleo.2811

ISSN : 2101-0420

Éditeur

SAMRA

Édition imprimée

Date de publication : 28 décembre 2014

Pagination : 233-245

ISSN : 1145-3370

\section{Référence électronique}

Joseba Rios-Garaizar et Diego Garate, «Actualisation de l'inventaire des pointes de type Isturitz de la région cantabrique », PALEO [En ligne], 25 | 2014, mis en ligne le 28 juillet 2015, consulté le 07 juillet 2020. URL : http://journals.openedition.org/paleo/2811; DOI : https://doi.org/10.4000/paleo.2811

\section{(c) $($ ) $९$}

PALEO est mis à disposition selon les termes de la licence Creative Commons Attribution - Pas d'Utilisation Commerciale - Pas de Modification 4.0 International. 


\title{
Actualisation de l'inventaire des pointes de type Isturitz de la région cantabrique
}

\author{
Joseba RIOS-GARAIZAR ${ }^{(1)}$, Diego GARATE(2)
}

\begin{abstract}
Résumé : Nous présentons dans ce travail une révision du corpus des pointes de type Isturitz du Gravettien cantabrique, concernant les exemplaires déjà connus de Bolinkoba, Usategi et Aitzbitarte III, ainsi que celui provenant de la récente découverte du site d'Askondo. L'analyse inclut une approche technologique et fonctionnelle de ces pointes grâce à laquelle nous pouvons conclure que deux types primaires sont représentés : les pointes fusiformes légères (Aitzbitarte III et Askondo) et les pointes triangulaires massives (Bolinkoba, Usategi). De plus, la distribution des sites possédant des pointes de type Isturitz dans la région cantabrique nous permet d'associer ces armatures avec celles des sites attribués au Gravettien riche en burins de Noailles. Ceci, avec d'autres éléments tels que la mobilité des matières premières, la technologie lithique ou les manifestations artistiques, nous permet d'y repérer une articulation territoriale particulière pendant le Gravettien dans cette région.
\end{abstract}

Mots-clés : Gravettien, pointe de type Isturitz, industrie osseuse, territorialité, région cantabrique.

Abstract: An updated inventory of the Isturitz type bone points of the cantabrian region. In this work we present a review of the Isturitz type bone point assemblage of the cantabrian Gravettian, which includes the already known pieces from Bolinkoba, Usategi and Aitzbitarte III, and also the point recently discovered in Askondo site. This review includes technological and use-wear analyses which helped to identify two major types: light fusiform points (Aitzbitarte III and Askondo) and massive triangular points (Bolinkoba and Usategi). Also, we noted that the spatial distribution of the Isturitz type points in the cantabrian region is similar to the distribution of the sites defined as Gravettian rich in Noailles burins. This evidence, combined with the available information about raw material mobility, the technological organization and the artistic manifestations, suggest a particular territorial articulation of this region during the Gravettian.

Key-words: Gravettian, Isturitz type point, Bone Industry, Territoriality, Cantabrian Region.

\section{Introduction}

Les pointes de type Isturitz sont l'un des outils les plus caractéristiques et représentatifs du Gravettien d'Europe occidentale (San Juan-Foucher et Vercoutère 2003). Les outils de ce type sont spécialement abondants dans la région des Pyrénées, associés au Gravettien de faciès Noailles (Foucher 2013 ; San Juan-Foucher 2013), bien qu'ils soient aussi présents en Dordogne (Goutas 2008 ; San Juan-Foucher et Vercoutère 2003) et peut être dans quelques sites du nord de la France (Goutas 2013). Dans la péninsule ibérique, ce type d'outillage osseux apparaît exclusivement dans la région cantabrique, plus concrètement à son extrémité orientale, où se concentrent les sites gravettiens riches en burins de Noailles, dont les plus importants sont, d'ouest en est, Bolinkoba, Antoliña,

(1)Centro Nacional de Investigación sobre la Evolución Humana (CENIEH). Paseo Sierra de Atapuerca, 3, ES-09002 Burgos joseba.rios@cenieh.es

(2)Arkeologi Museoa Calzadas de Mallona s/n, ES-48006 Bilbao - garatemaidagandiego@gmail.com 
Amalda et Aitzbitarte III. Ce dernier site montre des liens évidents, concernant la matière première, la technotypologie et sans doute l'économie de subsistance, avec le Sud-Ouest de la France, en particulier avec le site d'Isturitz situé à proximité (Altuna et al. 2011 ; Normand et al. 2013).

Le nombre des pointes de type d'Isturitz retrouvées dans la région cantabrique est très réduit (fig. 1). Jusqu'en 2010 , trois exemplaires avaient été identifiés, provenant de Bolinkoba, Aitzbitarte III et Usategi (Corchón 1986 ; Mujica 1983 ; San Juan-Foucher et Vercoutère 2003) auxquels il faut ajouter désormais le fragment de pointe découvert dans la grotte d'Askondo (Garate et Rios-Garaizar 2013). La distribution de ces pièces dans la région cantabrique coïncide avec celle du Gravettien riche en burins de Noailles Cette distribution nous permet d'envisager un lien entre les sites de l'extrémité orientale cantabrique et les Pyrénées occidentales (Isturitz et Aitzbitarte III en sont les plus emblématiques) et de les différencier de ceux de la zone cantabrique centrale et occidentale.

Un aspect notable des pointes de type Isturitz est l'homogénéité des procédés techniques utilisés pour leur fabrication et la variabilité restreinte des formes qui se limite à quelques types primaires (Goutas 2008). Leur fonction reste toutefois incertaine puisqu'il existe peu de données fonctionnelles directes, étant donné que la majorité des pointes conservent uniquement leur partie proximale. Ainsi, les inférences fonctionnelles ont été obtenues presque exclusivement à partir de la morphologie des pièces, de la position et de la nature de certains caractères particuliers telles que les stries dorsales et latérales ou les encoches.

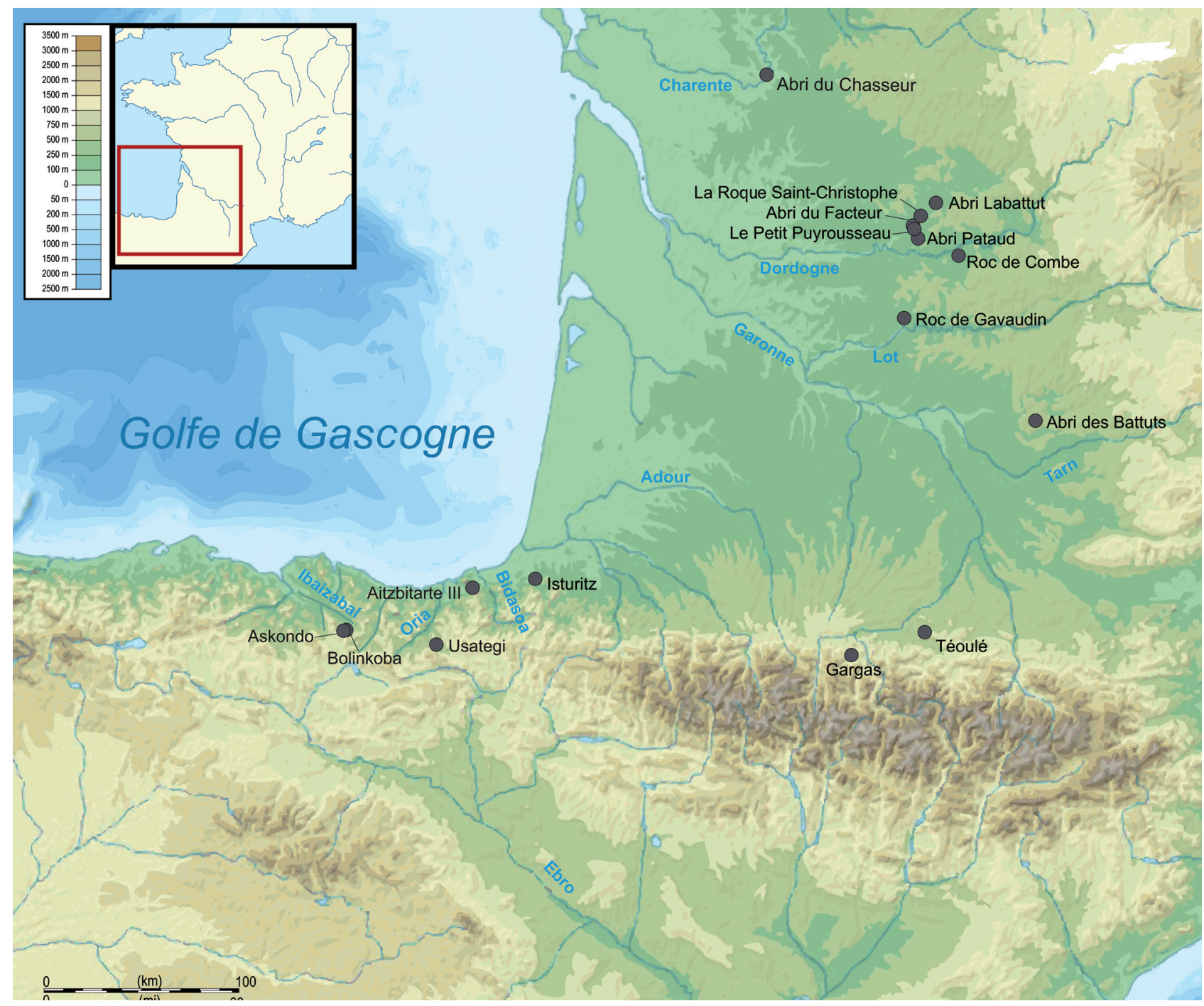

Figure 1 - Carte des sites avec pointes de type Isturitz autour du Golfe de Gascogne. D'après N. Goutas 2008. Fond de carte modifié à partir de l'original d'Eric Gaba.

Figure 1 - Map with the archaeological sites with Isturitz type points around the Bay of Biscay. Modified from N. Goutas 2008. Base cartography modified from the original of Eric Gaba. 
Au cours de cette étude, nous allons réaliser une description technologique et fonctionnelle des pointes de type Isturitz de la péninsule ibérique, en les mettant en relation avec le contexte archéologique dans lequel elles ont été localisées. Ceci nous permettra d'évaluer leur importance pour la compréhension du Gravettien dans la région cantabrique et les liens éventuels avec les sites des Pyrénées.

\section{1 - Matériels et Méthodes}

Pour décrire les objets, nous avons suivi la méthodologie proposée par N. Goutas (2008). Les pointes ont été analysées de visu et à l'aide de loupes binoculaires Olympus SZX1 et Nikon du Arkeologi Museoa - Musée d'Archéologie de Biscaye (Bilbao) et du Centre de Dépôt des Matériels Archéologiques de Gipuzkoa (SaintSébastien). De plus, nous fournissons les dimensions maximales des pointes ainsi que l'épaisseur et la largeur prises à des différentes distances de l'extrémité proximale pour réussir à représenter leur degré d'amincissement (tabl. 1). En outre, les caractères morphologiques et technologiques sont synthétisés dans le tableau 2.

\section{1 - Askondo}

II s'agit de la découverte la plus occidentale réalisée à ce jour, d'une pointe d'Isturitz. Elle fut trouvée en surface dans la grotte d'Askondo, située sur les abords du Parc Naturel d'Urkiola, dans les années 1980. Plus tard, en 2010, un ensemble important d'art «pré-magdalénien 》 a été retrouvé dans cette même grotte, daté de 23760 \pm 110 uncal BP $\left({ }^{14} \mathrm{C}\right.$ AMS, Beta -303671$)$ à partir de l'analyse ${ }^{14} \mathrm{C}$ AMS d'un os planté dans la paroi près d'un panneau décoré (Garate et Rios-Garaizar 2013). Par ailleurs, on a mis en évidence dans le vestibule de ce site, une séquence archéologique composée de niveaux du Paléolithique moyen, ainsi que de plusieurs niveaux datés entre 31000 et 17000 BP (Rios-Garaizar ; Garate, Regalado 2012). La pointe a été conservée dans le Hontza Museoa jusqu'en 2011 et se trouve actuellement à l'Arkeologi Museoa à Bilbao. L'état de conservation est plutôt bon, même si la pièce présente une fracture récente à l'une des extrémités et que sa surface est couverte de vernis, ce qui rend son observation plus difficile (fig. 2). Pour cette raison, la matière première de son support fut déterminée initialement comme de l'os (Rios-Garaizar et Mozota Holgueras 2012) puis, après un examen plus détaillé, comme du bois de cervidé.

La pointe est fusiforme, les bords étant parallèles sur la zone centrale et convergents sur la zone proximale. L'extrémité proximale appointée est légèrement déjetée par rapport à l'axe longitudinal de la pièce, créant ainsi une morphologie typique en biseau latéral. La section est biconvexe, les faces inférieures et supérieures convergent dans un angle de $80^{\circ}$.

Le mode de débitage du support démarre probablement par le rainurage du bois et, ensuite, le support a certainement été extrait par une fracture par flexion, même si nous ne voyons pas de traces techniques qui nous permettraient de l'affirmer.

Le façonnage du support a été réalisé par raclage de la face extérieure du bois, créant ainsi une surface relativement lisse (fig. $2 a$ ). Ce raclage est beaucoup plus intense et régulier sur les faces près des bords pour former un angle. Sur la face inférieure, le raclage est moins fort, de manière à préserver une partie du tissu spongieux. La pièce se termine par la réalisation des incisions caractéristiques de la face supérieure et des bords sur son tiers proximal (fig. 2c, b). Sur la face supérieure, les incisions sont peu profondes, fines et transversales à l'axe longitudinal. Sur les bords, les incisions sont plus profondes et plus courtes $(5-2,7 \mathrm{~mm})$ et ne sont pas réalisées à intervalles réguliers.

\section{2 - Bolinkoba}

Sur le site de Bolinkoba, situé dans le même massif montagneux qu'Askondo, un fragment proximal d'une pointe de type Isturitz a été trouvé dans le niveau F. Ce niveau présente une grande densité de matériels, avec 760 outils lithiques retouchés parmi lesquels dominent les burins, dont 50 sont du type de Noailles. On y retrouve également des pointes de La Gravette et des pointes des Vachons. L'industrie osseuse est elle aussi abondante, avec des esquilles appointées et des fragments de côtes décorées avec des séries d'incisions latérales, très comparables à celles d'Isturitz (San Juan-Foucher 2013). II y a aussi des éléments de parure réalisés sur des coquillages de Littorina et de Nassa reticulata (Garate 2012). La faune est composée essentiellement de bouquetins, mais la présence de carnivores est significative, parmi lesquels se trouvent le renard, la panthère, le loup et l'ours (Castaños 1983). La pointe est conservée actuellement dans les réserves de l'Arkeologi Museoa à Bilbao. L'état de conservation de la pièce, fabriquée en bois de cerf, est plutôt bon, même si elle présente plusieurs fractures récentes (fig. 3b), probablement produites au cours des fouilles, ainsi qu'une légère corrosion sur sa face supérieure (fig. 3c).

La morphologie de l'extrémité préservée de la pièce est triangulaire, les deux bords convergeant sur l'axe longitudinal, la pièce complète était très probablement fusiforme. La section est biconvexe et asymétrique, les faces supérieure et inférieure du bord gauche convergent en un biseau aigu, alors que le bord droit est chanfreiné.

La pièce présente des traces technologiques liées au processus d'extraction du support, principalement le rainurage (fig. 3j). Le façonnage de la pointe s'est fait par raclage en deux phases, une de raclage grossier et une deuxième de raclage plus fin ou d'abrasion qui efface presque totalement la première. Ce raclage concerne la totalité de la face supérieure et seulement de manière partielle la face inférieure, permettant ainsi qu'une partie du tissu spongieux du bois soit conservée. Postérieurement, des incisions ont été réalisées, des traits simples, parfois multiples, sur les bords et sur la face supérieure de la pièce, 


\begin{tabular}{|l|c|c|c|c|}
\cline { 2 - 5 } \multicolumn{1}{c|}{} & Askondo & Bolinkoba & Usategi & Aitzbitarte III \\
\hline Longueur & 96 & 84 & 84 & 142 \\
\hline Largeur & 25 & 29 & 27 & 25 \\
\hline Largeur à 2 cm de l'extrémité proximale & 13 & 14 & 16 & 14 \\
\hline Largeur à 4 cm de l'extrémité proximale & 20 & 23 & 22 & 17 \\
\hline Épaisseur & 10 & 12 & 19 & 10 \\
\hline Épaisseur à 2 cm de l'extrémité proximale & 9 & 9 & 12 & 8 \\
\hline Épaisseur à 4 cm de l'extrémité proximale & 10 & 11 & 14 & 9 \\
\hline Poids (en grammes) & 14.8 & 15.5 & 25.1 & 26.3 \\
\hline
\end{tabular}

Tableau 1 - Dimensions (en $\mathrm{mm}$ ) des pointes de type Isturitz analysées.

Table 1 - Measurements of analyzed Isturitz type points.

\begin{tabular}{|l|c|c|c|c|}
\cline { 2 - 5 } \multicolumn{1}{l|}{} & Askondo & Bolinkoba & Usategi & Aitzbitarte III \\
\hline Morphologie & & & & \\
\hline Fusiforme & $\mathrm{x}$ & & & $\mathrm{x}$ \\
\hline Triangulaire & & $\mathrm{x}$ & $\mathrm{x}$ & \\
\hline Pointe à méplat latéral & $\mathrm{x}$ & & & $\mathrm{x}$ \\
\hline Section & & & & \\
\hline Biconvexe & $\mathrm{x}$ & $\mathrm{x}$ & & $\mathrm{x}$ \\
\hline Ovalaire/Rectangulaire & & & $\mathrm{x}$ & \\
\hline Bords & & & & $\mathrm{x}$ \\
\hline Encoches latérales & $?$ & & $\mathrm{x}$ & \\
\hline Chanfrein gauche & & & $\mathrm{x}$ & \\
\hline Chanfrein droit & & $\mathrm{x}$ & $\mathrm{x}$ & \\
\hline Biseau & $\mathrm{x}$ & $\mathrm{x}$ & & $\mathrm{x}$ \\
\hline Incisions face & & & & \\
\hline supérieure & & & & $\mathrm{x}$ \\
\hline Série unique & & $\mathrm{x}$ & & $\mathrm{x}$ \\
\hline Série multiple & $?$ & & $\mathrm{x}$ & \\
\hline Incisions droit & & & & $\mathrm{x}$ \\
\hline Séparées & & $\mathrm{x}$ & & \\
\hline Superposées & & & & \\
\hline Série unique & & & & \\
\hline Série multiple & & & & \\
\hline Régulières & & & & \\
\hline Incisions gauche & & & & \\
\hline Séparées & & & & \\
\hline Superposées & & & & \\
\hline Série unique & & & & \\
\hline Série multiple & & & \\
\hline Régulières & & & & \\
\hline Fractures & & & & \\
\hline Distale en languette & & & & \\
\hline Proximale en languette & & & & \\
\hline Récente & & & & \\
\hline
\end{tabular}

Tableau 2 - Caractéristiques morphologiques et technologiques des pointes de type Isturitz analysées.

Table 2 - Morphological and technological features of analyzed Isturitz type points. 


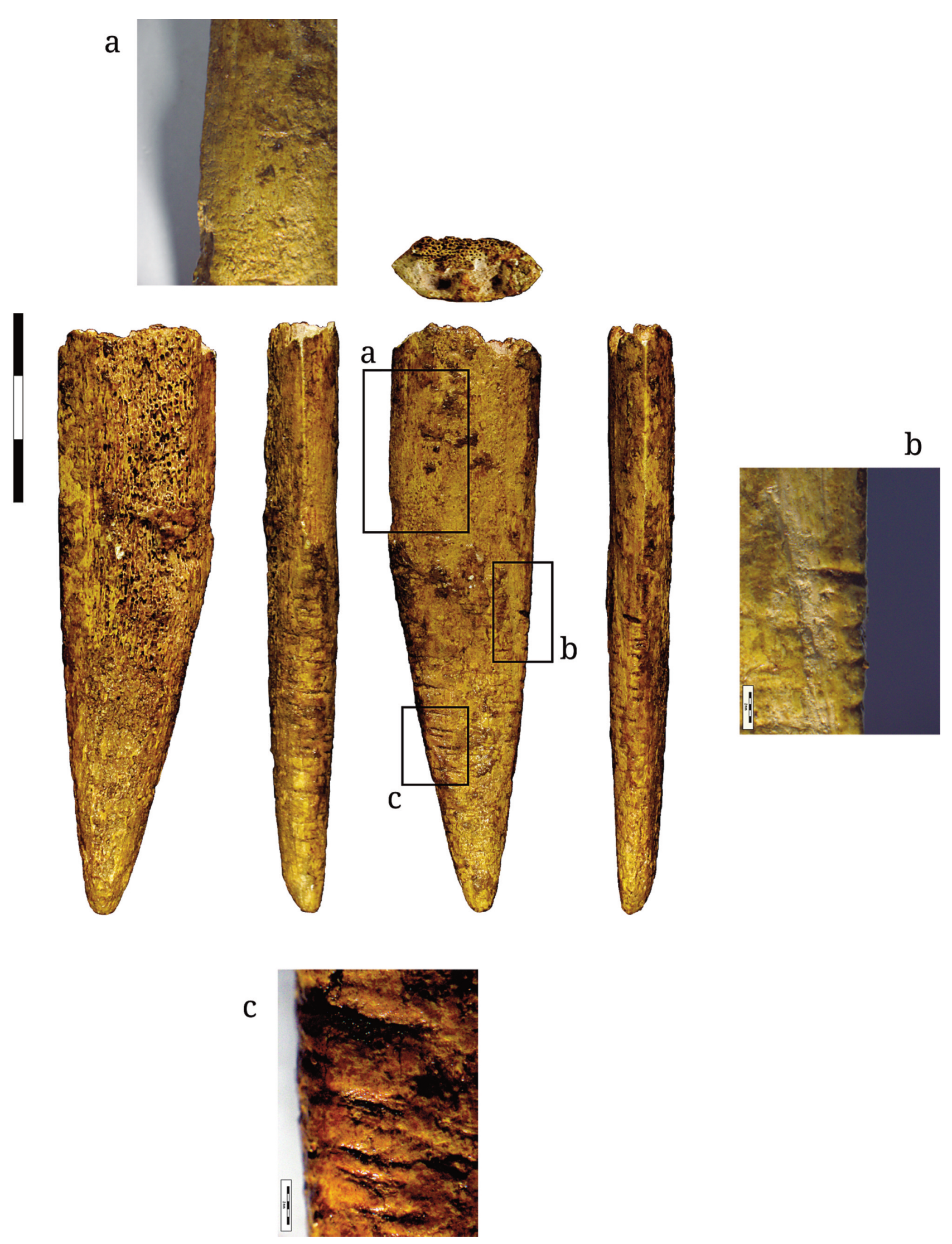

Figure 2 - Pointe de type Isturitz d'Askondo.

Figure 2 - Isturitz type point from Askondo. 

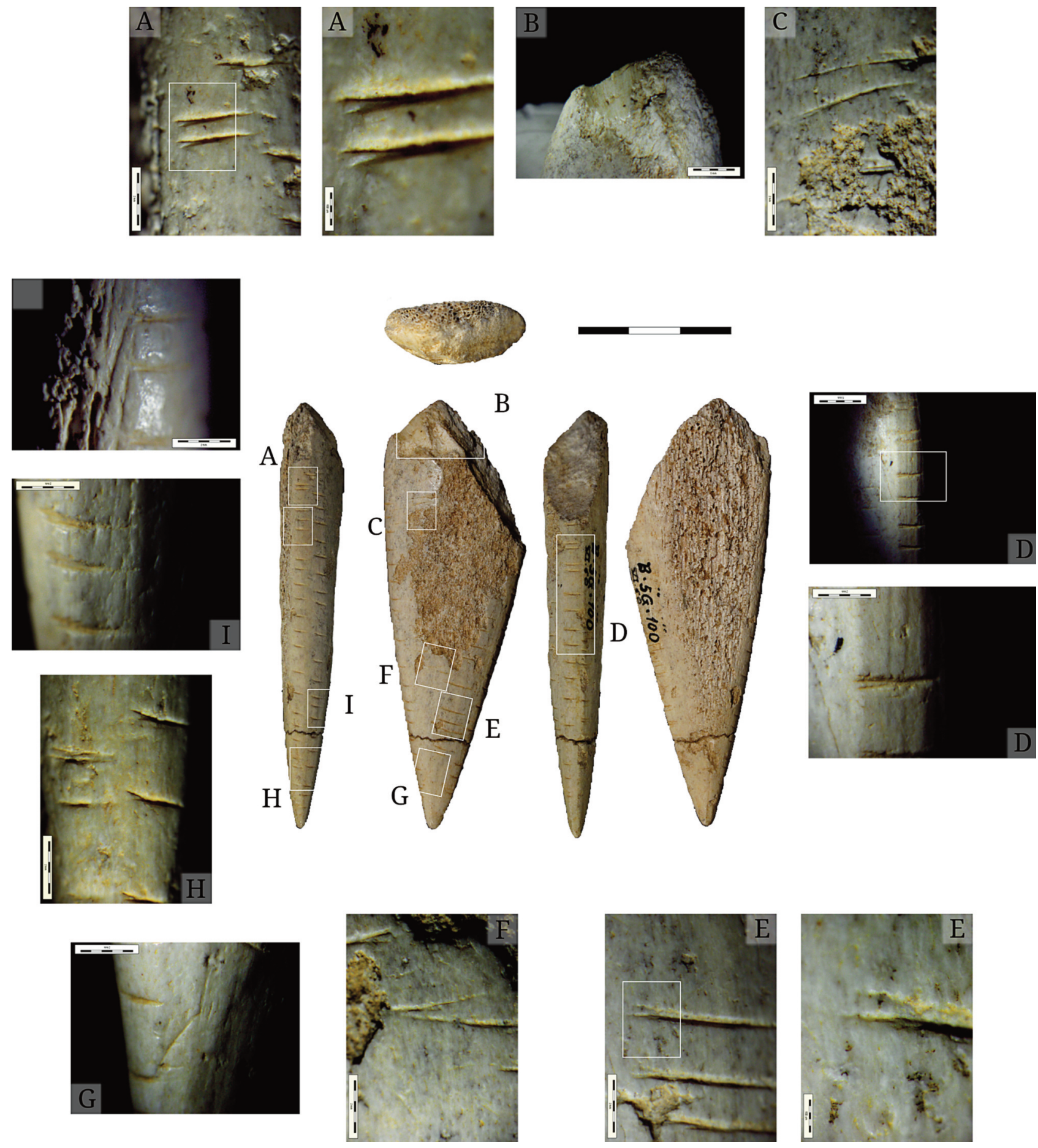

Figure 3 - Pointe de type Isturitz de Bolinkoba.

Figure 3 - Isturitz type point from Bolinkoba. 
concernent uniquement l'extrémité proximale. Des incisions parallèles, transversales à l'axe longitudinal de la pièce, ont étés réalisées sur la face supérieure, elles sont relativement profondes et courtes $(6 \mathrm{~mm})$ (fig. 3f, e). Sur la surface la plus éloignée de la pointe, ces incisions semblent plus fines et plus longues, mais la mauvaise conservation de cette partie nous empêche de l'affirmer de manière catégorique (fig. $3 \mathrm{c}$ ). Les incisions des latéraux des bords droit et gauche sont différentes. Sur le bord droit il s'agit d'incisions très courtes $(<3 \mathrm{~mm})$ réalisées à partir de la face inférieure et de la face supérieure, se disposant face à face (fig. $3 a, g, h, i)$. Sur le bord gauche, une série unique d'incisions profondes apparaît, réalisées à intervalles réguliers (fig. 3d). Sur la face ventrale, il n'y a pas d'incisions parallèles.

\section{3 - Usategi}

Le site de la grotte d'Usategi se trouve à la source de la vallée de l'Oria. Les fouilles réalisées par J. M. Barandiarán dans les années 1970 ont mis au jour une stratigraphie de cinq niveaux. Dans le niveau III, parmi quelques restes d'industrie lithique et osseuse, un fragment de pointe d'Isturitz a été découvert (Barandiarán 1977), conservé actuellement au Centre de Dépôt du Matériel Archéologique de Gipuzkoa à Saint-Sébastien. II s'agit d'un fragment proximal d'une pointe en bois de cervidé. La fracture est ancienne et concerne la partie mésio-proximale de la pièce. En plus de la fracture, la pièce montre une altération due à la corrosion de la face supérieure qui a éliminé une partie du tissu compact (fig. 4).

La forme de la pointe est sub-triangulaire. Les bords convergent sur l'axe longitudinal de la pièce. La section est rectangulaire à pans arrondis.

La pointe ne présente pas de traces techniques occasionnées par le processus d'extraction du support mais, par contre, on peut voir celles de son façonnage. En effet, on observe un raclage longitudinal réalisé par un outil lithique, intense sur la face dorsale (fig. $4 b$ ) et qui réduit à peine le tissu spongieux de la face inférieure. Suite à la mise en forme par raclage, les incisions transversales de la face supérieure ont été tracées à l'aide d'un outil lithique. Elles se distribuent de manière irrégulière sur la face supérieure, sont absentes sur les $12 \mathrm{~mm}$ de l'extrémité proximale et moins denses sur la zone proche de la fracture mésio-distale. Ces incisions s'organisent sur trois bandes longitudinales : elles sont presque perdues sur la bande de gauche à cause de l'altération de la face supérieure (fig. 4f), transversales sur la bande centrale (fig. 4b) et légèrement obliques sur la bande de droite (fig. $4 \mathrm{e}, \mathrm{c}$ ). Les premières qui ont été réalisées sont celles de la bande de droite, allant de droite à gauche et d'un seul trait. Celles de la bande centrale coupent ces dernières et ont été repassées, pour certaines d'entre elles, par un trait d'aller-retour (fig. 4c). Celles de la bande de gauche semblent couper celles de la bande centrale. Postérieurement, deux encoches ont été réalisées sur le bord droit (fig. 4d), coupant ainsi les incisions de la bande de droite de la face supérieure (fig. $4 \mathrm{~d}, \mathrm{e}$ ). L'encoche la plus proche de la pointe mesure
$13 \times 8 \mathrm{~mm}$, alors que la deuxième, qui semble plus grande, n'est conservée que sur une partie $(9 \times 7,5 \mathrm{~mm})$. Ces encoches ont été réalisées par entaillage avec un outil lithique, de la partie distale vers l'extrémité proximale. Cet entaillage génère un profil en $V$ asymétrique, l'inclinaison vers la partie proximale étant plus prononcée. Pour finir, le bord gauche a d'abord été chanfreiné par un raclage longitudinal, puis des incisions très profondes, transversales à l'axe longitudinal, ont été réalisées tout le long du bord, en suivant un tracé rectiligne, (fig. 4a). Les espaces entre les incisions ne sont pas réguliers, elles se superposent même les unes aux autres sur quelques points concrets, particulièrement sur la zone la plus proche de la fracture.

La pièce présente une fracture en languette écrasée et l'extrémité distale est légèrement polie, probablement par l'usure.

\section{4 - Aitzbitarte III}

Le site d'Aitzbitarte III se situe sur la colline du même nom, à l'extrémité orientale de la région cantabrique. Les fouilles qui ont eu lieu au cours des années 1980 et 1990 par J. Altuna ont mis au jour une intéressante séquence du Paléolithique supérieur initial (Altuna 2011). La pointe fut retrouvée dans le remplissage d'un conduit qui reliait Aitzbitarte III et Aitzbitarte II, qui contenait aussi une abondante faune et beaucoup de matériel lithique, dont plusieurs burins de Noailles (Mujica 1983). Ce remplissage fut interprété comme provenant de la zone intérieure de la première grotte où se trouvent les niveaux noailliens datés aux alentours de 24500 uncal BP (Altuna 1992). La pointe fut publiée par J.A Mujica (1983) et se trouve actuellement au Centre de Dépôt du Matériel Archéologique de Gipuzkoa. Elle a été réalisée à partir de bois de cerf et présente une fracture distale ancienne, une fracture proximale ancienne et une fracture moderne sur cette dernière. La pièce a été consolidée, le tissu spongieux en particulier, puisqu'elle était très fragile au moment de sa découverte (fig. 5).

La forme de la pointe est fusiforme, avec des bords qui sont parallèles jusqu'à $6,3 \mathrm{~cm}$ de l'extrémité proximale, là où ils commencent à converger de manière asymétrique, le bord droit présentant l'inclinaison la plus marquée, de telle façon que la pointe est déjetée à gauche de l'axe longitudinal. La section est convexe - concave. Sur l'extrémité mésiale, aux bords parallèles, le bord droit a un biseau plus marqué alors que celui du bord gauche est un peu plus arrondi. Vers l'extrémité proximale, les biseaux deviennent des méplats latéraux, le droit incliné vers la face supérieure et le gauche vers la face inférieure.

La pièce ne porte pas de traces techniques liées à l'extraction du support mais présente néanmoins d'abondants stigmates de façonnage. On peut lire sur la face supérieure deux phases différenciées de raclage, l'une plus grossière, probablement destinée à éliminer la surface rugueuse extérieure du bois, et une autre plus fine, peutêtre d'abrasion, pour la finition (fig. 5a, b). La face inférieure conserve le tissu spongieux. 

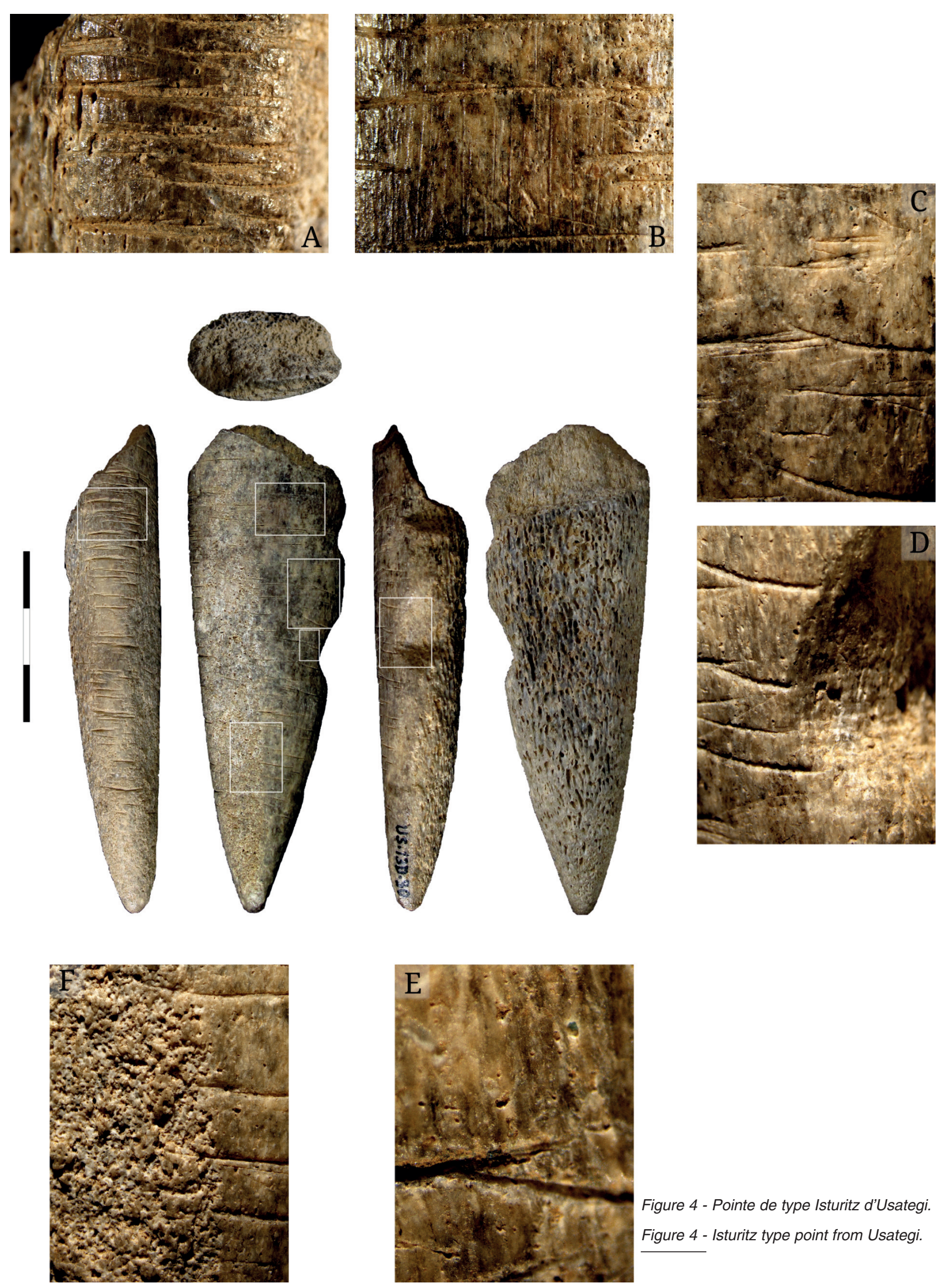

Figure 4 - Pointe de type Isturitz d'Usategi. Figure 4 - Isturitz type point from Usategi. 

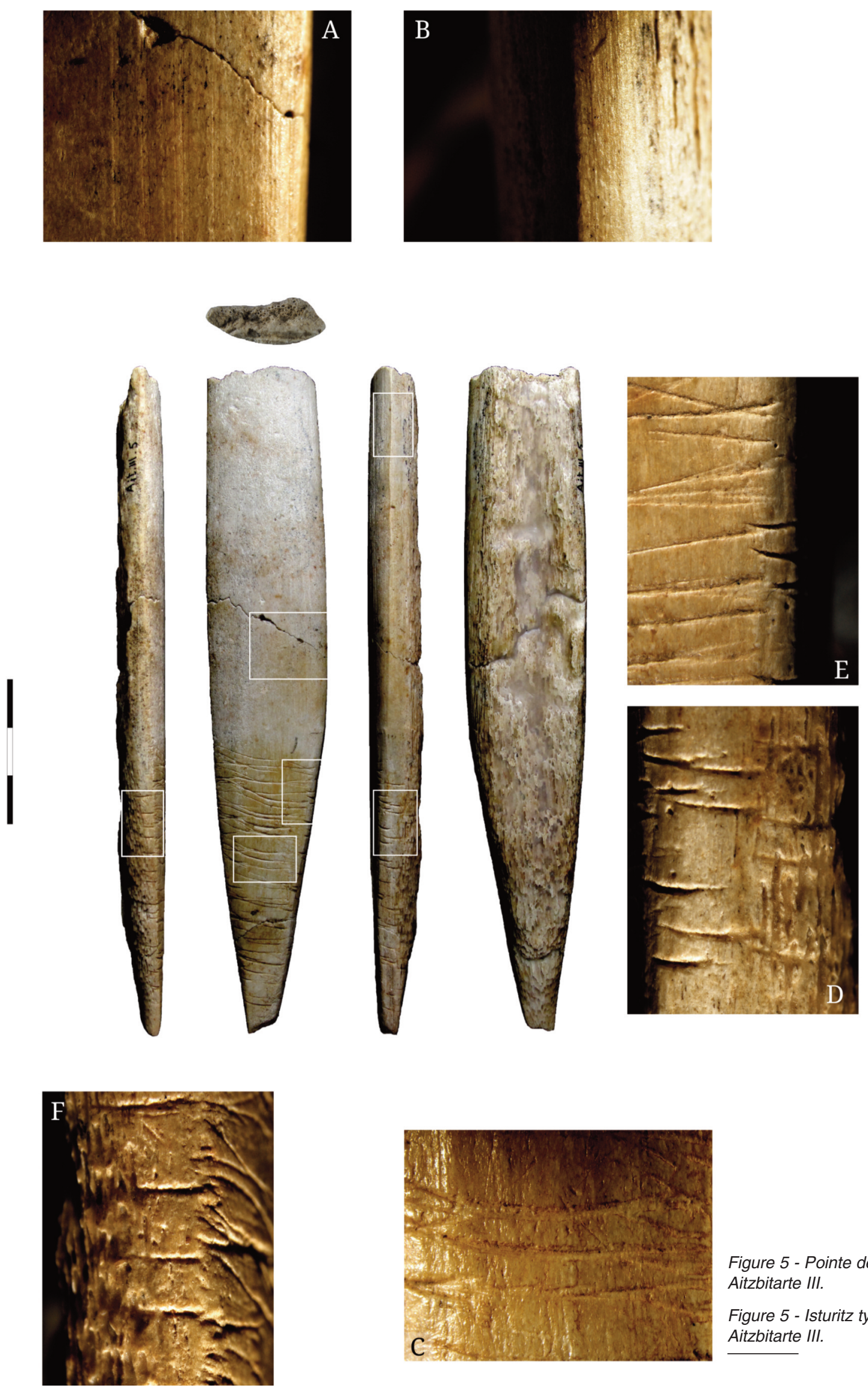

Figure 5 - Pointe de type Isturitz de Aitzbitarte III.

Figure 5 - Isturitz type point from Aitzbitarte III. 
Après le raclage, des incisions ont été réalisées sur la surface supérieure et sur les bords. Les incisions de la face supérieure et celles des bords commencent pratiquement sur l'extrémité proximale et s'arrêtent à $6,5 \mathrm{~cm}$ de la pointe, justement dans la partie mésiale de la pièce où les bords latéraux prennent une disposition parallèle. Les incisions de la face supérieure sont transversales et occupent toute la largeur de la face supérieure, pouvant même parfois se superposer (fig. $5 \mathrm{c}, \mathrm{e}$ ). Leur tracé est légèrement convexe vers l'extrémité proximale, elles sont faites de traits fins, uniques et peu profonds, réalisés de droite à gauche. De plus, d'autres traits plus courts et très peu profonds démarrent sur les bords à droite et à gauche, envahissant à peine la face supérieure (fig. 5e). Les incisions du bord droit sont multiples ; certaines, petites et courtes, commencent sur l'intersection avec la face supérieure, coupant les incisions de celle-ci ; d'autres, plus longues, recouvrent l'épaisseur du bord ; et finalement, d'autres encore, courtes elles aussi, commencent sur l'intersection avec la face inférieure (fig. 5d). Ces traits sont clairement individualisés, ne se superposant pas, et ne sont pas séparés par des espaces réguliers. Sur le bord gauche, on voit seulement une série de traits courts séparés par des intervalles réguliers (fig. $5 f$ ). La pièce présente une fracture distale en languette écrasée ainsi qu'une petite fracture proximale en languette.

\section{2 - Discussion}

La présence de pointes de type Isturitz dans la région cantabrique fut initialement reconnue par J.-M. Barandiarán, directeur des fouilles de Bolinkoba et d'Usategi, deux des sites où sont apparus ce type d'objets en contexte archéologique précis (Barandiarán 1977, 1978). Postérieurement, s'est ajoutée au répertoire la pointe d'Aitzbitarte III, site fouillé sous la direction de J. Altuna, mais cette fois sans un contexte archéologique clair (Mujica 1983). C'est autour de ces mêmes dates que l'on retrouva la pointe d'Askondo, même si elle ne fut identifiée en tant que telle et étudiée de manière préliminaire qu'en 2011 (Rios-Garaizar et Mozota Holgueras 2012).

Même si elles ont quelques traits en commun avec les pointes de type Isturitz, les deux pointes récupérées au niveau G (Gravettien) du site asturien de Cueto de la Mina (De la Rasilla et Santamaria 2006) ne peuvent être classifiées comme telles. Les différences sont multiples. L'extrémité proximale est pointue dans le cas des pointes d'Isturitz et arrondie pour celles de Cueto de la Mina. Aucun de ces deux exemplaires ne présente les formes typiques des pointes de type Isturitz, bien fusiformes ou bien triangulaires. De plus, les sections sont circulaires et en aucun cas aplaties. Le tissu spongieux de l'os ou du bois de cerf n'est pas préservé et pour finir, même si elles présentent des incisions sur la partie proximale, elles sont très profondes et leur disposition est différente de celle des pointes de type Isturitz. Un autre exemplaire remarquable est le fragment proximal de pointe récupéré au niveau XI (Magdalénien Inférieur) de la grotte de Las Caldas (Corchón 1994) également aux Asturies. Réalisé sur os, il remplit toutes les conditions formelles et techniques requises (forme pointue, section aplanie - quadrangulaire - incisions multiples et fines sur la face supérieure et sur les bords) pour être classé comme pointe de type Isturitz. Cependant, sa présence dans un niveau magdalénien est difficile à expliquer puisqu'il s'agit d'un exemplaire unique de ce type d'outil récupéré dans un contexte archéologique clairement non Gravettien. Enfin, sur le site d'Antoliñako Koba, la présence de fragments de sagaie sur bois de cerf à section ovalaire qui ressemblent aux pointes de type Isturitz a été signalée mais ils ne portent pas les incisions typiques sur la partie proximale (Aguirre 2013, fig. 4 : 1-5), incisions que l'on retrouve pourtant sur la base d'une sagaie aplatie (Aguirre 2013, fig. $4: 6$ ).

Avec ces éléments, on peut affirmer que les quatre pointes présentées ici sont les seules de la région cantabrique qui s'ajustent parfaitement aux morphologies requises pour les pointes de type Isturitz et qui ont été trouvées directement dans des contextes gravettiens (Aitzbitarte III et Bolinkoba). Dans le cas d'Askondo, la pointe est associée à un contexte rupestre et archéologique gravettien mais le contexte archéologique n'est pas évident. Finalement, dans le cas d'Usategi, l'attribution de l'ensemble au Gravettien se fait à partir de la présence de la pointe de type Isturitz, sans qu'il n'existe d'autres éléments archéologiques qui valident cette attribution.

Comme il a été démontré lors de travaux de révision de ce type d'objets (Goutas 2008 ; San Juan-Foucher et Vercoutère 2003 ; San Juan-Foucher 2013), les pointes de type Isturitz montrent une variabilité formelle relative, autant pour la morphologie des pièces que pour les types de section ou pour la disposition des incisions dorsales et latérales. Les quatre pointes récupérées sur les sites cantabriques démontrent aussi une certaine variabilité, autant au niveau morphométrique que d'un point de vue morphologique. Nous avons des exemplaires de plan fusiforme (Aitzbitarte III-Al et Askondo-AZ) ou triangulaire (Bolinkoba-B et Usategi- U), avec la pointe déjetée (Al, AZ) ou centrée $(B, U)$; avec des sections biconvexes / ovalaires $(\mathrm{Al}, \mathrm{AZ}, \mathrm{B})$ ou rectangulaires à pans arrondis $(U)$, et avec les bords chanfreinés $(B, U)$ ou biseautés $(A I, A Z, B)$. Les incisions de la face supérieure peuvent être uniques $(B)$, ou s'organiser en bandes (AZ, U, Al). Celles des bords peuvent être séparées, superposées, en séries uniques ou multiples et peuvent être réalisées à intervalles réguliers ou irréguliers et il peut y avoir, sur la même pièce, des différences entre le type d'incisions présentes sur les deux bords (tabl. 2). Du point de vue morphométrique, les pointes fusiformes (AI, AZ) rentrent dans la largeur standard des pointes du site d'Isturitz, alors que les deux triangulaires sont proches des valeurs maximales. Les dimensions d'épaisseur sont elles aussi proches des valeurs moyennes d'Isturitz (9,9 mm) (Goutas 2008), sauf dans le cas d'Usategi dont l'épaisseur de $19 \mathrm{~mm}$ dépasse largement cette moyenne.

Malgré le fait que la variabilité au sein des fragments de pointes analysés est grande, elle n'est pas aussi accentuée que dans le site d'Isturitz. II est donc difficile d'utiliser le 
classement employé par N. Goutas (2008), surtout si l'on tient compte de l'absence des fragments distaux. Le seul classement possible dans ce contexte serait de distinguer les pièces fusiformes et légères ( $A l, A Z)$ des pièces triangulaires et massives $(B, U)$.

En ce qui concerne la fonction de ces outils, l'apport de ces quatre pièces est limité. Deux d'entre elles présentent des fractures en languette écrasée qui peuvent être liées à une utilisation de pointes de projectiles ( $\mathrm{Al}, \mathrm{U})$, alors que les deux autres présentent des fractures récentes sur la partie proximale qui rendent plus difficile l'interprétation fonctionnelle (AZ, B). Il est important de remarquer l'absence de courbure sur l'axe longitudinal des pièces, qui peut être liée à une configuration favorable à leur utilisation comme des pointes de projectile.

En relation avec le contexte des pièces, il faut signaler que Aitzbitarte III et Bolinkoba ont livré des occupations gravettiennes intenses avec des burins de Noailles, des objets d'ornement et une industrie osseuse à caractère " domestique ». Ces deux sites se trouvent dans des paysages escarpés, dominant des zones de vallée, et ils ont des caractéristiques qui permettraient de les définir comme des lieux d'habitation. Cependant, si l'on compare avec Isturitz, il semble que ces deux sites ont un statut fonctionnel moins important. Les cas d'Usategi et d'Askondo sont différents. À Usategi, le contexte archéologique dans lequel la pointe est apparue est beaucoup plus pauvre et peu caractéristique, alors qu'il semblerait que la grotte d'Askondo n'ait été occupée pendant le Gravettien que pour la décoration de l'ensemble d'art pariétal.

\section{3 - Conclusions}

L'existence de ces pointes de type Isturitz dans la partie orientale de la région cantabrique est un élément complémentaire à d'autres indices comme la circulation des matières premières ou l'unité techno-typologique des industries lithiques (Tarriño 2011 ; Rios-Garaizar et al. 2011 ; Foucher et al. 2012 ; Arrizabalaga et De la Peña 2013 ; Foucher 2013 ; San Juan-Foucher 2013 ; Normand, de la Peña, San Emeterio 2013 ; Arrizabalaga et al. 2014) qui renforcent l'impression d'une certaine unité culturelle du Gravettien dans la région des Pyrénées, incluant la partie orientale de la région cantabrique. Les sites du Gravettien riches en Noailles des provinces de Biscaye et Gipuzkoa semblent très liés au site d'Isturitz mais, par rapport à ce site, ils présentent toujours un statut fonctionnel mineur si l'on considère l'extension et l'intensité des occupations, l'abondance des industries lithique et osseuse, y compris les pointes de type Isturitz, et l'abondance des éléments de parure et des pièces d'art mobilier (Normand et al. 2013 ; Garate, Rivero 2014).

En revanche, une analyse récente des liens existants entre l'art pariétal de chronologie gravettienne (thèmes, techniques et ressources expressives) de ces régions, et contrairement à ce qui est déduit, des ensembles industriels, a montré des liens plus forts au sein de la région cantabrique pendant le Gravettien, qu'entre le Sud-Ouest de la France et la partie orientale de la région cantabrique (Garate et al. sous presse).

Les avancées significatives sur la connaissance du Gravettien de la région cantabrique, réalisées dans les dernières décennies, permettent une meilleure compréhension du techno-complexe gravettien ainsi que de ses relations avec d'autres régions voisines. Cette modeste contribution permet d'établir de manière plus précise le rôle des pointes de type Isturitz dans cette discussion.

\section{Remerciements}

Nous voulons remercier les responsables du Centre de Dépôt du Matériel Archéologique de Gipuzkoa et du Musée d'Archéologie de Biscaye pour les facilités accordées pour l'étude du matériel archéologique présenté dans cet article. La traduction du manuscrit espagnol a été faite par Pauline Buthaud. Nous voulons remercier aussi les relecteurs de cet article et spécialement Cristina San Juan-Foucher pour leurs commentaires et les corrections apportées sur le manuscrit original.

\section{Références bibliographiques}

AGUIRRE M. 2013 - Ocupaciones gravetienses de Antoliñako koba: aproximación preliminar a su estratigrafía, cronología e industrias. In : C. de las Heras, J. A. Lasheras, A. Arrizabalaga, M. De la Rasilla (Eds.), Pensando El Gravetiense: Nuevos Datos Para La Región Cantábrica En Su Contexto Peninsular Y Pirenaico. Madrid, Ministerio de Educación, Cultura, p. 216-228 (Monografías Del Museo Nacional Y Centro de Investigación de Altamira, № 23).

ALTUNA J. 1992 - Dataciones de radiocarbono para el Perigordiense Superior del País Vasco. Munibe 43, p. 31-32.

ALTUNA J. 2011 - Las cuevas de Aitzbitarte (Landarbaso, Rentería). Situación e historia de las investigaciones. In : J. Altuna, K. Mariezkurrena, J. Rios-Garaizar (Eds.), Ocupaciones Humanas En La Cueva de Aitzbitarte III (Renteria, País Vasco) Sector Entrada: 33.000-18.000 BP. Vitoria-Gasteiz, Eusko Jaurlaritzaren Argitalpen Zerbitzu Nagusia, p. 11-21.

ALTUNA J., MARIEZKURRENA K., RIOS-GARAIZAR J. 2011 - Ocupaciones Humanas en la Cueva de Aitzbitarte III (Renteria, País Vasco) Sector Entrada 33 000-18 000 B. Vitoria-Gasteiz, Eusko Jaurlaritzen Argitalpen Zerbitzu Nagusia.

ARRIZABALAGA A., DE LA PEÑA P. 2013 - El registro de la industria lítica como base para la organización del Gravetiense cantábrico. In : C. de las Heras, J. A. Lasheras, A. Arrizabalaga, M. De la Rasilla (Eds.), Pensando El Gravetiense: Nuevos Datos Para La Región 
Cantábrica En Su Contexto Peninsular Y Pirenaico. Madrid, Ministerio de Educación, Cultura, p. 367-386 (Monografías Del Museo Nacional Y Centro de Investigación de Altamira, № 23).

ARRIZABALAGA A., CALVO A., ELORRIETA I., TAPIA J., TARRIÑO A. 2014 - Where To and What For? Mobility Patterns and the Management of Lithic Resources by Gravettian Hunter-Gatherers in the Western Pyrenees. Journal of Anthropological Research, 70, p. 233-261.

BARANDIARÁN J.M. 1977 - Excavaciones en Jentilbaratza y Kobalde (Ataun) (Campaña de 1971). Munibe 29, p. 195-212.

BARANDIARÁN J.M. 1978 - Bolinkoba y otros yacimientos prehistóricos de la Sierra de Amboto. In : J. M. Barandiarán (Ed.), Obras Completas de José Miguel de Barandiaran Tomo XII. Bilbao, La Gran Enciclopedia Vasca, p. 393-453.

CASTAÑOS P. 1983 - Estudio de los macromamíferos del yacimiento prehistórico de Bolinkoba. Kobie 13, p. 261-298.

CORCHÓN M. S. 1986 - El arte mueble paleolítico cantábrico: contexto y análisis interno. Madrid, Subdirección General de Arqueología y Etnografía.

CORCHÓN M. S. 1994 - Arte mobiliar e industria ósea solutrense en la Cornisa Cantábrica. Férvedes 1, p. 131-148.

DE LA RASILLA M., SANTAMARÍA D. 2006 - El Gravetiense del Abrigo de Cueto de la Mina (Posada de Llanes, Asturias). Zona Arqueológica, Miscelánea en Homenaje a Victoria Cabrera 1, p. 422-439.

FOUCHER P. 2013 - Synthèse chrono-culturelle sur le Gravettien des Pyrénées: constat et réflexions sur la stabilité régionale des traditions techniques. $I n$ : C. de las Heras, J. A. Lasheras, A. Arrizabalaga, M. De la Rasilla (Eds.), Pensando El Gravetiense: Nuevos Datos Para La Región Cantábrica En Su Contexto Peninsular Y Pirenaico. Madrid, Ministerio de Educación, Cultura, p. 151-168 (Monografías Del Museo Nacional $Y$ Centro de Investigación de Altamira, № 23).

FOUCHER P., SAN JUAN-FOUCHER C., VERCOUTĖRE C., FERRIER C. 2012. La grotte de Gargas (Hautes-Pyrénées, France) : l'apport du contexte archéologique à l'interprétation de l'art pariétal. In J. Clottes (dir) L'art pléistocène dans le monde / Pleistocene art of the world / Arte pleistoceno en el mundo. Actes du Congrès IFRAO, Tarascon-sur-Ariège, 6-11 septembre 2010. $N^{\circ}$ spécial de Préhistoire, Art et Sociétés, Bulletin de la Société Préhistorique Ariège-Pyrénées, LXV-LXVI, 20102011, livre : p. 52-53 et CD : p. 209-225.

GARATE D. 2012 - Neandertales y Cromañones. Los primeros pobladores de Bizkaia. Guías del Arkeologi Museoa, 2. Bilbao, Diputación Foral de Bizkaia, Bizkaikoa y Arkeologi Museoa.
GARATE D., RIOS-GARAIZAR J. 2013 - El arte parietal paleolítico de la cueva de Askondo (Mañaria, Bizkaia. In : C. de las Heras, J. A. Lasheras, A. Arrizabalaga, M. De la Rasilla (Eds.), Pensando El Gravetiense: Nuevos Datos Para La Región Cantábrica En Su Contexto Peninsular Y Pirenaico. Madrid, Ministerio de Educación, Cultura, p. 512525 (Monografías Del Museo Nacional Y Centro de Investigación de Altamira, № 23).

GARATE D., RIVERO O. 2014 - L'art mobilier gravettien sur support lithique de la grotte d'Isturitz (Saint-Martind'Arberoue, Pyrénées-Atlantiques) : une collection redécouverte. Paleo $n^{\circ} 25$, p. 247-276

GARATE D., RIVERO O., RUIZ-REDONDO A., RIOSGARAIZAR J. sous presse - In the crossroads: New findings of rock art around the Biscay Bassin for the understanding of paleolithic symbolic expression. Quatenary International.

GOUTAS N. 2008 - Les pointes d'Isturitz sont-elles toutes des pointes de projectile? Gallia Préhistoire 50, p. 45-101.

GOUTAS N. 2013 - Nouvelles données sur l'industrie osseuse du gravettien des grottes d'Arcy-sur-Cure (Yonne, France): vers l'identification de nouveaux marqueurs techniques et culturels du Gravettien moyen à burins du Raysse. In : P. Bodu, L. Chehmana, L. Klaric, L. Mevel, S. Soriano, N. Teyssandier (Eds.), Actes de La Table Ronde Internationale: Le Paléolithique Supérieur Ancien de l'Europe Du Nord-Ouest. Séance de La Société Préhistorique Française (15-18 Avril 2009, Musée de Sens, Yonne). Paris, Societé Préhistorique Française, p. 89-115.

MUJICA J. A. 1983 - Industria de hueso en la prehistoria de Gipúzcoa. Munibe 35, p. 451-631.

NORMAND C., GOUTAS N., LACARRIERE J., SIMONET A. 2013 - El Gravetiense de Isturitz : nuevas investigaciones, nuevos datos. In : C. de las Heras, J. A. Lasheras, A. Arrizabalaga, M. De la Rasilla (Eds.), Pensando El Gravetiense: Nuevos Datos Para La Región Cantábrica En Su Contexto Peninsular Y Pirenaico. Madrid, Ministerio de Educación, Cultura, p. 171-194 (Monografías Del Museo Nacional Y Centro de Investigación de Altamira, № 23).

RIOS-GARAIZAR J., MOZOTA M. 2012 - Estudio de la industria lítica, ósea y cerámica del yacimiento de Askondo (Mañaria, Bizkaia). In : D. Garate, J. Rios-Garaizar (Eds.), La Cueva de Askondo (Mañaria, Bizkaia). Arte Parietal Y Ocupación Humana Durante La Prehistoria. Kobie. Bilbao, Diputación Foral de Bizkaia, p. 77-88. (Serie BAl, 2).

RIOS-GARAIZAR J., DE LA PEÑA P., SAN EMETERIO A. 2011 - Estudio de las industrias líticas y óseas de la cueva de Aitzbitarte III (Zona de la entrada). In : Altuna, J., Mariezkurrena, K., Rios-Garaizar, J. (Eds.), Ocupaciones Humanas En La Cueva de Aitzbitarte III (Renteria, País Vasco) Sector Entrada: 33.000-18.000 BP. Eusko Jaurlaritzaren Argitalpen Zerbitzu Nagusia, Vitoria-Gasteiz, p. 81-351. 
RIOS-GARAIZAR J., GARATE D., REGALADO E. 2012 Excavación arqueológica en la cueva de Askondo (Mañaria, Bizkaia). In : D. Garate, J. Rios-Garaizar (Eds.), La Cueva de Askondo (Mañaria, Bizkaia). Arte Parietal Y Ocupación Humana Durante La Prehistoria. Kobie. Bilbao, Diputación Foral de Bizkaia, p. 31-38. (Serie BAI, 2).

SAN JUAN-FOUCHER C. 2013 - Industria ósea decorada y arte mueble del Gravetiense Pirenaicoriales actualizadas. In : C. de las Heras, J. A. Lasheras, A. Arrizabalaga, M. De la Rasilla (Eds.), Pensando El Gravetiense: Nuevos Datos Para La Región Cantábrica En Su Contexto Peninsular $Y$ Pirenaico. Madrid, Ministerio de Educación, Cultura, p. 461-483 (Monografías Del Museo Nacional Y Centro de Investigación de Altamira, № 23).
SAN JUAN-FOUCHER C., VERCOUTÈRE C. 2003 - Les " sagaies d'Isturitz » des niveaux gravettiens de Gargas (Hautes-Pyrénées) et de Pataud (Dordogne). Préhistoires Méditerranéennes 12, p. 75-94.

TARRIÑO A. 2011 - Procedencia de los sílex de la cueva de Aitzbitarte III (Rentería, Gipuzkoa). In : J. Altuna, K. Mariezkurrena, J. Rios-Garaizar, J. (Eds.), Ocupaciones Humanas En La Cueva de Aitzbitarte III (Renteria, País Vasco) Sector Entrada: 33 000-18 000 BP. Eusko Jaurlaritzaren Argitalpen Zerbitzu Nagusia, Vitoria-Gasteiz, p. 353-373. 
